\title{
Development of fully depleted CCD imager NCUcam-1 and follow-up observations for PS1 sky surveys
}

\section{Daisuke Kinoshita}

National Central University, Taiwan

email: kinoshita@astro.ncu.edu.tw

\begin{abstract}
Visible 4-color simultaneous imager is being developed for a new 2-m telescope at Lulin observatory in Taiwan. The main task of this instrument is quick and efficient followup observations for large scale surveys, such as PS1 sky surveys. NCUcam-1 is one of unit cameras for this instrument. It is equipped with a new type of CCD chip, fully depleted CCD, and have superb sensitivity at the wavelength of 1 micron. Igneous asteroids exhibit prominent absorption feature around 1 micron due to minerals, and NCUcam-1 is able to deliver reliable taxonomic classifications. NCUcam-1 has achieved the first-light using 1-m telescope at Lulin in July 2011. Results of the characterization work for NCUcam-1 and tests observations of asteroids are reported. Upcoming plan for the study of pair asteroids search and identification is also presented.
\end{abstract}

